Article

\title{
Long-Term Weight Change after Initiating Second-Generation Antidepressants
}

\author{
David Arterburn ${ }^{1, *},+$, Tamar Sofer ${ }^{2, \dagger}$, Denise M. Boudreau ${ }^{1, \dagger}$, Andy Bogart ${ }^{1, \dagger}$, \\ Emily O. Westbrook ${ }^{1,+}$, Mary Kay Theis ${ }^{1,+}$, Greg Simon ${ }^{1,+}$ and Sebastien Haneuse ${ }^{3,+}$ \\ 1 Group Health Research Institute, 1730 Minor Ave, Suite 1600, Seattle, WA 98101, USA; \\ boudreau.d@ghc.org (D.M.B.); abogart@rand.org (A.B.); westbrook.e@ghc.org (E.O.W.); \\ theis.m@ghc.org (M.K.T.); simon.g@ghc.org (G.S.) \\ 2 University of Washington, UW Tower, 15th Floor, 4333 Brooklyn Ave NE, Seattle, WA 98105, USA; \\ tsofer@uw.edu \\ 3 Harvard T.H. Chan School of Public Health, 677 Huntington Ave, Boston, MA 02115, USA; \\ shaneuse@hsph.harvard.edu \\ * Correspondance: arterburn.d@ghc.org; Tel.: +1-206-287-4610 \\ + These authors contributed equally to this work.
}

Academic Editor: Nuri B. Farber

Received: 31 December 2015; Accepted: 6 April 2016; Published: 13 April 2016

\begin{abstract}
Objective: To examine the relationship between the choice of second-generation antidepressant drug treatment and long-term weight change; (2) Methods: We conducted a retrospective cohort study to investigate the relationship between choice of antidepressant medication and weight change at two years among adult patients with a new antidepressant treatment episode between January, 2006 and October, 2009 in a large health system in Washington State. Medication use, encounters, diagnoses, height, and weight were collected from electronic databases. We modeled change in weight and BMI at two years after initiation of treatment using inverse probability weighted linear regression models that adjusted for potential confounders. Fluoxetine was the reference treatment; (3) Results: In intent-to-treat analyses, non-smokers who initiated bupropion treatment on average lost $7.1 \mathrm{lbs}$ compared to fluoxetine users who were non-smokers (95\% CI: $-11.3,-2.8$; $p$-value $<0.01)$; smokers who initiated bupropion treatment gained on average $2.2 \mathrm{lbs}$ compared to fluoxetine users who were smokers (95\% CI: $-2.3,6.8$; $p$-value $=0.33)$. Changes in weight associated with all other antidepressant medications were not significantly different than fluoxetine, except for sertraline users, who gained an average of $5.9 \mathrm{lbs}$ compared to fluoxetine users (95\% CI: 0.8 , 10.9; $p$-value $=0.02)$; (4) Conclusion: Antidepressant drug therapy is significantly associated with long-term weight change at two years. Bupropion may be considered as the first-line drug of choice for overweight and obese patients unless there are other existing contraindications.
\end{abstract}

Keywords: obesity; antidepressant; depression; adverse effects; weight gain

\section{Introduction}

Obesity and depression are major public health concerns with substantial impacts on medical morbidity, health care spending, and quality of life [1-7]. In 2011-2012, the prevalence of obesity (defined as a body mass index [BMI] of $\geqslant 30 \mathrm{~kg} / \mathrm{m}^{2}$ ) among US adults was 34.9\% [8], and, in 2008, $10.4 \%$ of US adults were taking antidepressant medications [9]. Obesity and depression also commonly occur together, and adults with both conditions may have even greater health risks [10-12]. The causal pathway is probably bidirectional-obese adults are at greater risk of depression and vice versa.

With climbing rates of obesity $[13,14]$ and antidepressant agents now the most commonly prescribed drugs in the US [15], the potential impact of antidepressant use on obesity risk is receiving 
renewed attention [16]. There is now a growing body of evidence that the choice of antidepressant drug therapy may influence changes in weight $[17,18]$. Previous studies indicate that certain antidepressants (e.g., fluoxetine and bupropion) may cause weight loss, while others (e.g., paroxetine and mirtazapine) may cause weight gain, although follow-up data is generally limited to the first 12 months after treatment [18-24]. Less evidence is available regarding the long-term impact of antidepressants on weight, and some associations appear to be transient [18-23]. Still, many patients are prescribed antidepressants for long time periods, and it is important to know whether this longer-term exposure is related to weight gain.

Second-generation antidepressants (i.e., selective serotonin reuptake inhibitors (SSRIs), serotonin norepinephrine reuptake inhibitors (SNRIs), and selective serotonin norepinephrine reuptake inhibitors (SSNRIs)) are the most commonly prescribed medications for primary treatment of depression, and they have similar efficacy and lower toxicity in overdose than first-generation antidepressants [25]. Current evidence indicates that second-generation antidepressants do not differ from each other on the basis of efficacy $[26,27]$. Therefore, treatment guidelines suggest that clinicians should select antidepressants on the basis of adverse effect profiles, costs, and patient preferences [25]. If antidepressants differ significantly in their long-term impact on weight, then patients and clinicians could preferentially choose those medications that are associated with the least amount of weight gain when prescribing them for patients who are normal weight, overweight, or obese. Thus, having more information on the long-term impact of antidepressants on weight could reduce the downstream risk of weight-related morbidity for a large population of adults with depression.

The goal of this study was to examine the relationship between the choice of antidepressant drug treatment and long-term weight gain at the population level. Because one of the most commonly prescribed antidepressants, bupropion, is also used as an adjunct to smoking cessation, and smoking cessation is strongly associated with weight gain [28], we sought to examine the effects of this antidepressant on weight gain among smokers and non-smokers separately. To accomplish these goals, we conducted a retrospective electronic health record (EHR)-based cohort study of patients initiating a monotherapy second-generation antidepressant (hereafter referred to as "antidepressant") drug treatment between 2005 and 2009.

\section{Experimental Section}

\subsection{Setting}

The study was conducted at Group Health (GH), an integrated health plan and care delivery system that provides comprehensive health care to approximately 600,000 individuals in Washington State and Idaho. Among these enrollees, 65\% receive care at GH Integrated Group Practice (IGP) clinics while the remaining enrollees are allied with non-GH provider networks where the primary source of data is the claims system. Information on health plan enrollment and health care use including diagnoses, procedures, pharmacy dispensings, and laboratory values are recorded and maintained in GH's automated electronic databases. Further, since 2005, a fully-integrated EHR system documents all patient care at GH IGP clinics.

GH provides specialty mental health care to its enrollees using a combined IGP/network model. GH guidelines emphasize cognitive-behavioral therapy as part of first-line therapy for depressive disorders; however, antidepressant drug treatment surpasses psychotherapy as the initial treatment of choice for depression at GH, and 75\% of antidepressant therapy is prescribed by Primary Care Physicians (PCPs).

The GH population closely resembles the underlying Washington state community with respect to age, race, and gender [29]. GH insurance plans vary considerably in the level of cost-sharing for outpatient primary care and mental health care, but copayments for routine outpatient care and psychotherapy are similar to national averages. Prior studies suggest that GH enrollees obtain approximately $97 \%$ of their prescription medications at GH owned and contracted pharmacies [29-31]. 


\subsection{Study Population}

The study population consists of adults aged 18-65 years with a diagnosis of depressive disorder (International Classification of Diseases, 9th Revision, ICD-9: 296.2×, 296.3×, 311, or 300.4) and who initiated a new monotherapy episode of antidepressant drug treatment. The latter was defined as a dispensing episode for a single antidepressant medication, without any other antidepressant medication dispensing in the prior nine months. The date of initiation/prescription is referred to as the "baseline date", Our study sample was restricted to subjects who initiated the new treatment episode between 1 January, 2006 and 31 October, 2009, and had at least nine months of continuous enrollment in the health system prior to their baseline date.

Patients were excluded if they were taking medications or underwent procedures that have a strong effect on weight change. These included second/third-generation antipsychotic medications, lithium, valproate, weight loss medications, oral steroids, bariatric surgery, and dialysis. A full list of all excluded medications is provided in Table S1. Similarly, patients with histories of cirrhosis, eating disorder, pregnancy, or dementia at baseline were excluded from our analyses based on the presence of diagnosis codes.

\subsection{Data Collection}

Demographic and enrollment information, weight and height, prescription medication use, health care encounters, and medical conditions were extracted from GH electronic health care databases for all years of our study.

\subsection{Antidepressant Drug Treatment}

We extracted all fills for second-generation antidepressant medications, the primary exposure of interest, from 04/2005-09/2010. These included fluoxetine, citalopram, bupropion, paroxetine, sertraline, trazodone, mirtazapine, venlafaxine, and duloxetine. During the years of our study the second-generation antidepressant medications ecitalopram, fluvoxamine, and nefazodone were not on the Group Health formulary. As a result these three medications were rarely prescribed and were excluded from our analyses due to low sample sizes. A full list of all included and excluded medications is provided in Table S1.

\subsection{Weight Data}

The primary outcome of interest was the change in weight between the start of the new treatment episode (baseline) and two years post-baseline. Weight data was extracted from all encounters from 04/2005-09/2010. Encounters were classified according to several criteria: (i) occurring within the IGP vs. with a network provider; (ii) ambulatory (i.e., in-person, outpatient) encounter vs. other encounter type (emergency room and inpatient); and (iii) primary care vs. specialty visit. In the GH IGP, care standards indicate that weights should be obtained at each outpatient visit without extra clothing and shoes. Prior research at GH indicates that weight measures routinely obtained in clinical care are highly correlated with those obtained by trained research staff and may be used in research studies without statistical correction [32].

To account for missingness in the weight data, we adopted a strategy that combined imputation prior to the main statistical analyses with inverse probability weighting in the main statistical analyses (see below). Prior to imputation we applied a novel cleaning algorithm that removed implausible measurements (i.e., those outside $50-500 \mathrm{lbs}$.) as well as measurements that were inconsistent with an individual's weight trajectory. Briefly, this algorithm consisted of fitting an unadjusted linear model of weight as a function of time to each subject's weight data separately, and excluding those measurements for which the standardized residual was greater than 3 , provided the subject had enough weight measurements to estimate such a model. For subjects with too few weight measurements to fit the linear model, no measurements were excluded. 
At baseline, we adopted the following sequential imputation strategy for weight: (i) if weight information was available on the baseline date, we took that value; (ii) otherwise, if a weight measurement was observed in the 30 days prior to the baseline date, we carried that value forward; (iii) otherwise, weight was left as missing. At the two year follow-up time point (i.e., day 730), we adopted a similar strategy: (i) if weight information was available on day 730 we took that value; (ii) otherwise, if a weight measurement was observed in the +/ -15 days around day 730 , we carried the nearest value backward/forward; (iii) if there were at least two weight measurements, at least 30 days apart, in a 180 day interval around day 730 (i.e., + / -90 days), we fit an individual-specific linear regression model to these values and interpolated to obtain a weight at day 730; (iv) otherwise, weight was left as missing. The requirement of at least two weight measurements being at least 30 days apart was set in order to prevent unstable weight extrapolation.

\subsection{Covariates}

Potential confounders of the relationship between choice of antidepressant drug treatment and weight change in time, obtained from electronic health care databases, were age, gender, having history of anxiety disorder, bipolar disorder, sleep disorder, schizophrenia and schizoaffective disorders, and smoking status at the time of initiating antidepressant treatment. The analyses were adjusted for these covariates' status at baseline.

\subsection{Statistical Analysis}

Distributions of patient characteristics were summarized by tabulating across levels and computing percentages; continuous covariates, such as age and baseline weight, were categorized for these descriptive purposes but retained in their continuous form for modeling. These initial descriptive analyses were conducted across all unique patients in the study sample. For patients with multiple treatment episodes, a single episode was chosen at random for these descriptive analyses.

To investigate the relationship between choice of antidepressant medication and weight change at two years we performed two sets of linear regression analyses. The first was based on the intent-to-treat (ITT) principle with outcomes at two years investigated purely on the basis of the medication prescribed at baseline (i.e., regardless of subsequent treatment discontinuation and/or modification), and was $a$ priori identified as our primary analysis. The second set was based on the per-protocol (PP) principle that considers the scenario in which patients continue their assigned antidepressant treatment for the full two years.

For all analyses, fluoxetine was taken as the "referent" medication comparator (or reference group). In addition to evaluating main effects, we investigated possible effect modification of bupropion use by smoking status, since bupropion is indicated for smoking cessation and smoking cessation is known to be associated with weight gain [28]. We hypothesized a priori that the impact of bupropion would differ by smoking status.

Throughout, estimates of regression parameters were obtained using inverse-probability weighting [33]. For the ITT analyses, the weights were developed to account for missing weight data at baseline and/or at two years. Specifically, we considered a patient as having "complete" data if a valid weight measurement was observed in the EHR both at baseline and at two years; a patient had "incomplete" data if either or both of these measurements was missing. We then estimated a patient's probability of having complete data as a function of risk factors observed in the EHR. Towards this, we developed a framework based on decomposing whether or not a patient had complete data into four sub-mechanisms: (i) a weight measurement was observed/imputed at baseline; (ii) the patient was continuously enrolled in the health plan until two years post-treatment initiation; (iii) a visit at an IGP clinic occurred at two years (+/ -1 month) and; (iv) a weight measurement was observed/imputed at 24 months. For each of these mechanisms, a separate regression model was fit; fitted values from the four models were multiplied to provide the overall probability of complete data. For the second of these mechanisms (i.e., continuously enrolled at 2 years) we modeled 
time-to-disenrollment using a Cox regression model; doing so permitted the use of information from patients who were administratively censored during follow-up. For the other three mechanisms, we modeled the appropriate binary outcome using logistic regression. For the PP analyses, these weights were combined with a separate set of weights, the inverse of the probability of treatment adherence throughout the two year follow-up. These probabilities were obtained from a Cox regression, which modeled time to treatment discontinuation; we obtained the probability of a patient to adhere to treatment throughout the two year follow-up. Finally, we note that all weights were truncated at the 90th percentile of their distributions to ensure stability [34]. While our primary results are based on these weighted analyses, for the sake of comparison, we also report unweighted analyses.

To ensure valid inference, we used the sandwich estimator [35] of the standard errors to account for the estimation of the inverse probability weights. Furthermore, to account for within-patient correlation (among those patients with more than one episode), we used a multiple outputation procedure [36]. Briefly, we developed multiple data sets of independent observations by selecting a single observation at random within each cluster/patient. We analyzed each resulting dataset, and combined the estimated regression parameters and standard errors according to a formula.

Throughout, all analyses were performed in R version 3.1.1. All $p$-values and confidence intervals were two-sided with statistical significance judged at $\alpha=0.05$ level.

\section{Results}

\subsection{Population Description}

Applying our inclusion/exclusion criteria, we identified 5932 patients who initiated 6186 monotherapy antidepressant treatment episodes during the study period. Of the 6186 episodes, 1017 episodes (among 969 patients) had complete baseline weight data and two-year weight data (Figure 1A); 229 episodes (among 967 patients) had complete weight data at both time points and had remained on treatment for the full two years (Figure 1B).

From Table 1 we see that the male-to-female ratios in the ITT and PP populations are similar to that of the entire population (approximately $67 \%-70 \%$ female). In addition, older patients were more likely to have complete weight data at two years (43\% of the ITT population was 50-65 years of age compared to $36 \%$ in the entire population) and also to have completed two years on treatment (50\% were 50-65 years of age). Similarly, the heaviest patients (220-500 lbs) were more frequently in the ITT and PP populations. Smokers comprised $32 \%$ of the population of treatment initiators, while only $29 \%$ and $22 \%$ of the ITT and PP populations, respectively.

Finally, fluoxetine users were $48 \%$ of treatment initiators, and tended to be more frequently represented in both the ITT and PP populations (52\% and 56\%, respectively). Users of other antidepressants had slight variations in representation rates across these population groups; bupropion users were slightly less frequently represented in the ITT and PP populations than in the treatment initiators. 


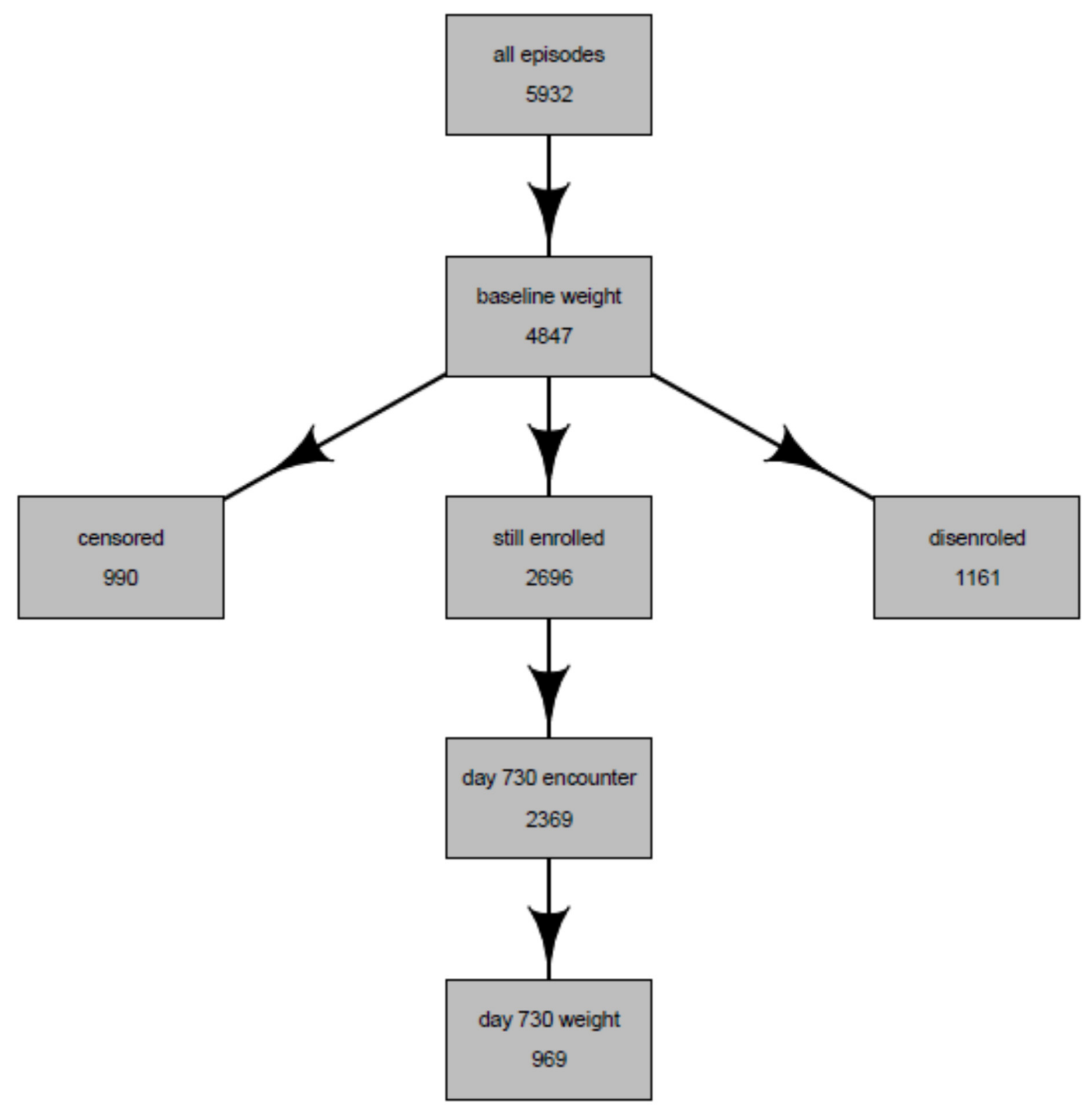

(A)

Figure 1. Cont. 


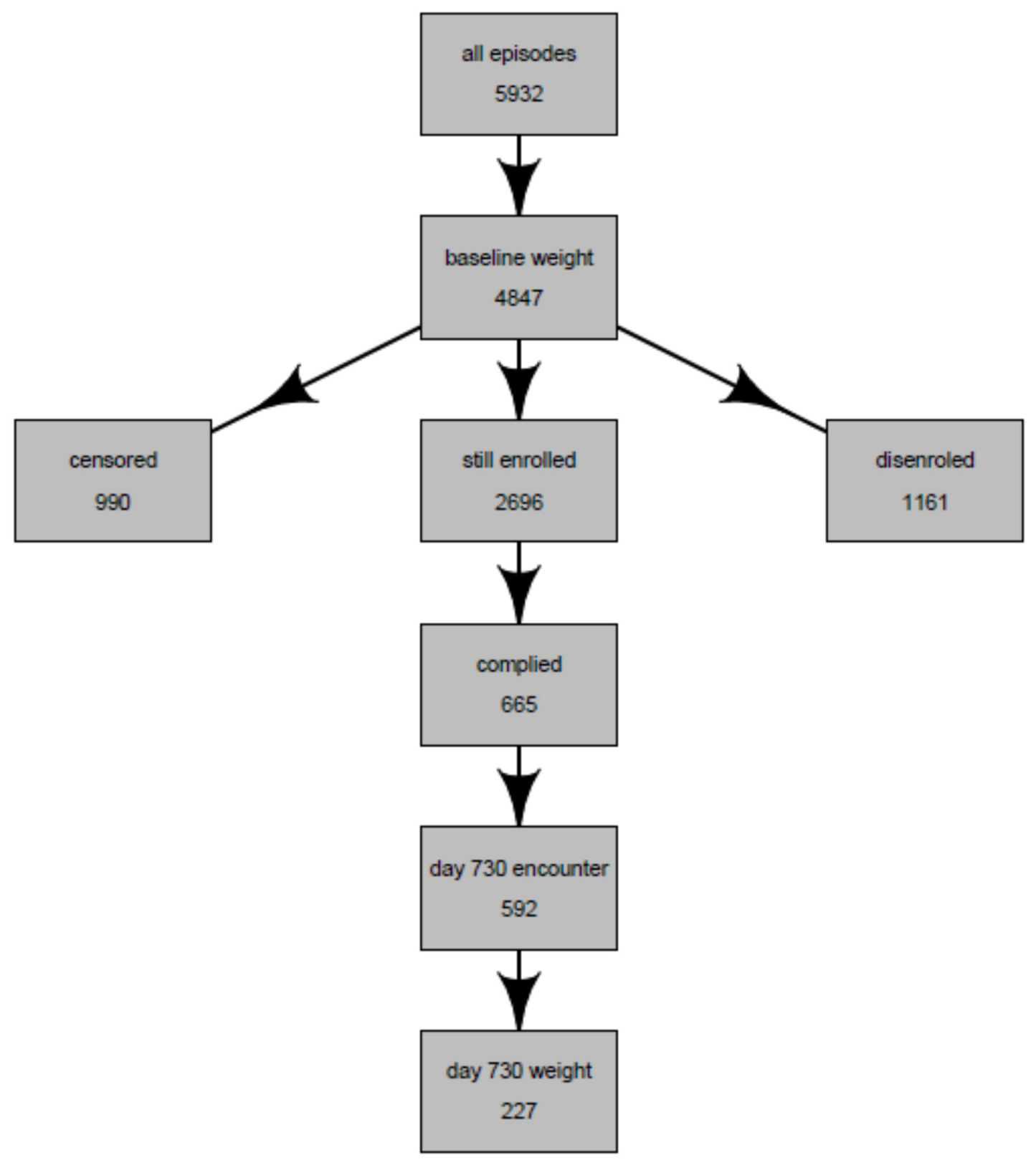

(B)

Figure 1. (A) Flow of the intent-to-treat population of antidepressant users; and (B) Flow of the per-protocol population of antidepressant users. Censored $=$ patients were censored if they were using drugs or underwent procedures that have strong effect on weight change (see methods); Still enrolled = still enrolled at Group Health at day 730 (two years); Day 730 encounter = patient had an ambulatory encounter in the Integrated Group Practice (IGP); Day 730 weight = patient had a weight recorded at that encounter. 
Table 1. Characteristics of the entire population of antidepressant users and antidepressant users with observable weight data at two years *.

\begin{tabular}{|c|c|c|c|}
\hline & $\begin{array}{l}\text { Entire Population of } \\
\text { Antidepressant Users * }\end{array}$ & $\begin{array}{l}\text { Population Whose } \\
\text { Weight Was Observed } \\
\text { at } 2 \text { years } \\
\text { (Intent-to-Treat } \\
\text { Population) }\end{array}$ & $\begin{array}{c}\text { Population Whose weight Was } \\
\text { Observed and Were } \\
\text { Continuously Treated with the } \\
\text { Same Antidepressant at } 2 \text { years } \\
\text { (Per-Protocol Population) }\end{array}$ \\
\hline Total & 5932 & 969 & 227 \\
\hline Female, $N(\%)$ & $4018(67.7 \%)$ & $651(67.2 \%)$ & $158(69.6 \%)$ \\
\hline \multicolumn{4}{|l|}{ Age, $N(\%)$} \\
\hline $18-29$ years & $1114(18.8 \%)$ & $119(12.3 \%)$ & $17(7.5 \%)$ \\
\hline $30-49$ years & $2691(45.4 \%)$ & $438(45.2 \%)$ & $96(42.3 \%)$ \\
\hline $50-65$ years & $2127(35.9 \%)$ & $412(42.5 \%)$ & $114(50.2 \%)$ \\
\hline \multicolumn{4}{|l|}{ Weight, $N(\%)$} \\
\hline 88-149 lbs. & $1176(19.8 \%)$ & $200(20.6 \%)$ & $42(18.5 \%)$ \\
\hline 150-179 lbs. & $1111(18.7 \%)$ & $226(23.3 \%)$ & $57(25.1 \%)$ \\
\hline 180-219 lbs. & $724(12.2 \%)$ & $148(15.3 \%)$ & $32(14.1 \%)$ \\
\hline 220-500 lbs. & $1188(20.0 \%)$ & $268(27.7 \%)$ & $69(30.4 \%)$ \\
\hline No baseline weight & $1085(18.3 \%)$ & $0(0 \%)$ & $0(0 \%)$ \\
\hline \multicolumn{4}{|l|}{ Body mass index, $N(\%)$} \\
\hline Underweight: <18.5 & $41(0.7 \%)$ & $9(0.9 \%)$ & $1(0.4 \%)$ \\
\hline Normal: $18.5-24.9$ & $1217(20.5 \%)$ & $216(22.3 \%)$ & $46(20.3 \%)$ \\
\hline Overweight: $25-29.9$ & $1324(22.3 \%)$ & $279(28.8 \%)$ & $66(29.1 \%)$ \\
\hline Obese: $\geqslant 30.0$ & $1957(33.0 \%)$ & $441(45.5 \%)$ & $110(48.5 \%)$ \\
\hline No baseline BMI & $1393(23.5 \%)$ & $24(2.5 \%)$ & $4(1.8 \%)$ \\
\hline \multicolumn{4}{|l|}{ Antidepressants, $N(\%)$} \\
\hline Fluoxetine & $2842(47.9 \%)$ & $506(52.2 \%)$ & $127(55.9 \%)$ \\
\hline Bupropion & $877(14.8 \%)$ & $129(13.3 \%)$ & $25(11 \%)$ \\
\hline Citalopram & $1137(19.2 \%)$ & $173(17.9 \%)$ & $39(17.2 \%)$ \\
\hline Duloxetine & $37(0.6 \%)$ & $8(0.8 \%)$ & $0(0.0 \%)$ \\
\hline Mirtazapine & $36(0.6 \%)$ & $5(0.5 \%)$ & $1(0.4 \%)$ \\
\hline Paroxetine & $245(4.1 \%)$ & $34(3.5 \%)$ & $9(4 \%)$ \\
\hline Sertraline & $367(6.2 \%)$ & $47(4.9 \%)$ & $18(7.9 \%)$ \\
\hline Trazodone & $281(4.7 \%)$ & $54(5.6 \%)$ & $6(2.6 \%)$ \\
\hline Venlafaxine & $110(1.9 \%)$ & $13(1.3 \%)$ & $2(0.9 \%)$ \\
\hline $\begin{array}{l}\text { Concurrent psychotherapy } \\
N(\%)\end{array}$ & $680(11.5 \%)$ & $100(10.3 \%)$ & $21(9.3 \%)$ \\
\hline \multicolumn{4}{|l|}{ Comorbid conditions, $N(\%)$} \\
\hline Anxiety disorder & $1527(25.7 \%)$ & $247(25.5 \%)$ & $49(21.6 \%)$ \\
\hline Bipolar disorder & $78(1.3 \%)$ & $9(0.9 \%)$ & $4(1.8 \%)$ \\
\hline Schizophrenia & $1(0.0 \%)$ & $0(0.0 \%)$ & $0(0.0 \%)$ \\
\hline Schizoaffective disorder & $1(0.0 \%)$ & $0(0.0 \%)$ & $0(0.0 \%)$ \\
\hline Sleep disturbance & $381(6.4 \%)$ & $80(8.3 \%)$ & $18(7.9 \%)$ \\
\hline Smoker & $1923(32.4 \%)$ & $282(29.1 \%)$ & $49(21.6 \%)$ \\
\hline
\end{tabular}

* The first column corresponds to characteristics of the entire population who had at least one monotherapy antidepressant treatment episode during the study period; the second column describes the characteristics of patients who were observed for weight change at two years, and were used in the intent-to-treat (ITT) analysis. The third column describes the characteristics of patients whose treatment episodes lasted at least two years, and were used in the per-protocol (PP) analysis. All covariates are measured at baseline.

\subsection{Intent-to-Treat Analysis Results}

Table 2 presents results of unweighted and inverse probability weighted ITT linear regression analyses of two-year weight change. Bupropion was the only drug that yielded a significantly different estimate of two-year weight loss when compared to fluoxetine, and then, only among patients who were non-smokers. In weighted analyses, non-smokers who initiated bupropion treatment experienced a weight change of $-7.1 \mathrm{lbs}$ compared to fluoxetine users who were non-smokers ( $95 \% \mathrm{CI}:-11.3$, $-2.8 ; p$-value $<0.01$ ). In contrast, smokers who initiated bupropion treatment gained, on average, an estimated $2.2 \mathrm{lbs}$ compared to fluoxetine users who were non-smokers (95\% CI: $-2.3,6.8 ; p$-value $=0.33$ ). Mirtazapine initiators gained, on average, an estimated $11.6 \mathrm{lbs}$ compared to fluoxetine users, although 
this difference was not statistically significant $(95 \% \mathrm{CI}:-2.8,26.0 ; p$-value $=0.12)$ likely due to the small number of mirtazapine treatment initiators in the data. All other antidepressant drug weight change estimates were not significantly different from fluoxetine, except for sertraline users, who gained 5.9 lbs compared to fluoxetine users (95\% CI: 0.8, 10.9; $p$-value $=0.02)$.

Table 2. Estimated 2-year weight change (lbs) for users of the various drug groups compared to fluoxetine users based on the intent-to-treat analysis *.

\begin{tabular}{ccccccc}
\hline & \multicolumn{3}{c}{ Unweighted Estimates } & \multicolumn{3}{c}{ Weighted Estimates } \\
\hline & Estimate & $\boldsymbol{p}$-Value & $\mathbf{9 5 \%} \mathbf{C I}$ & Estimate & $\boldsymbol{p}$-Value & $\mathbf{9 5 \%} \mathbf{~ C I}$ \\
\hline Bupropion-non smoker & -7.6 & $<0.01$ & $(-11.5,-3.7)$ & -7.1 & $<0.01$ & $(-11.3,-2.8)$ \\
Bupropion-smoker & 1.0 & 0.65 & $(-3.2,5.2)$ & 2.2 & 0.33 & $(-2.3,6.8)$ \\
Citalopram & 0.3 & 0.82 & $(-2.3,2.9)$ & 1.2 & 0.40 & $(-1.6,4.1)$ \\
Duloxetine & -0.6 & 0.91 & $(-11.4,10.1)$ & -1.0 & 0.88 & $(-13.5,11.5)$ \\
Mirtazapine & 12.7 & 0.08 & $(-1.5,27.0)$ & 11.6 & 0.12 & $(-2.8,26.0)$ \\
Paroxetine & -0.5 & 0.84 & $(-5.7,4.7)$ & 0.8 & 0.78 & $(-5.0,6.6)$ \\
Sertraline & 3.3 & 0.15 & $(-1.2,7.9)$ & 5.9 & 0.02 & $(0.8,10.9)$ \\
Trazodone & 0.4 & 0.84 & $(-3.9,4.8)$ & 0.8 & 0.75 & $(-3.9,5.5)$ \\
Venlafaxine & -6.7 & 0.14 & $(-15.5,2.1)$ & -2.0 & 0.67 & $(-11.3,7.3)$ \\
\hline
\end{tabular}

* Results in the left part of the table refer to the unweighted modelling that ignores selection bias, while the right side of the tables provides the inverse probability weighted (IPW) estimation results. Omnibus $p$-values for the null hypothesis "all drugs have the same effect on weight change" were 0.004 (naive analysis) and 0.009 (IPW analysis). Analyses were adjusted for age, gender, baseline weight, smoking status, and active psychotherapy.

Table 3 presents the mean baseline weight and BMI as well as the change in weight and BMI for each antidepressant medication in our study based on our ITT and IPW analysis. The mean baseline weight ranged from a low of $151.9 \mathrm{lbs}$ for mirtazapine and a high of $199.1 \mathrm{lbs}$ for non-smokers receiving bupropion. The mean baseline BMI ranged from $24.2 \mathrm{~kg} / \mathrm{m}^{2}$ for mirtazapine to $31.5 \mathrm{~kg} / \mathrm{m}^{2}$ for non-smokers receiving bupropion. The mean weight change at two years among the fluoxetine non-smokers was $+4.6 \mathrm{lbs}$, while there was mean weight loss of $2.4 \mathrm{lbs}$ among bupropion non-smokers. Bupropion smokers gained only slightly more weight (6.9 lbs) than fluoxetine smokers (6.7 lbs).

Table 3. Estimated baseline weight and body mass index (BMI) and change in weight and BMI at 2 years*.

\begin{tabular}{|c|c|c|c|c|}
\hline & $\begin{array}{c}\text { Baseline } \\
\text { Weight (lbs) }\end{array}$ & $\begin{array}{c}\text { Baseline BMI } \\
\left(\mathrm{kg} / \mathrm{m}^{2}\right)\end{array}$ & $\begin{array}{l}\text { Change in Weight } \\
\text { at } 2 \text { years (lbs) }\end{array}$ & $\begin{array}{c}\text { Change in BMI at } \\
2 \text { years }\left(\mathrm{kg} / \mathrm{m}^{2}\right)\end{array}$ \\
\hline Fluoxetine: non-smoker & 191.4 & 30.6 & 4.6 & 0.7 \\
\hline Fluoxetine: smoker & 186.2 & 29.5 & 6.7 & 1.1 \\
\hline Bupropion: non-smoker & 199.1 & 31.5 & -2.4 & -0.4 \\
\hline Bupropion: smoker & 194.0 & 30.2 & 6.9 & 1.1 \\
\hline Citalopram & 186.6 & 29.8 & 5.9 & 0.9 \\
\hline Duloxetine & 194.5 & 31.1 & 3.6 & 0.6 \\
\hline Mirtazapine & 151.9 & 24.2 & 16.2 & 2.6 \\
\hline Paroxetine & 189.9 & 30.1 & 5.5 & 0.9 \\
\hline Sertraline & 187.5 & 30.0 & 10.5 & 1.7 \\
\hline Trazodone & 188.1 & 29.8 & 5.4 & 0.9 \\
\hline Venlafaxine & 183.6 & 29.1 & 2.6 & 0.4 \\
\hline
\end{tabular}

* Results from the inverse probability weighted, intent-to-treat modeling analysis adjusted for age, gender, baseline weight, smoking status, and concurrent psychotherapy.

\subsection{Per Protocol Analysis Results}

Table 4 presents the results of both unweighted and IPW PP analysis. In our weighted analyses, bupropion was the only drug that yielded a significantly different two-year weight change compared with fluoxetine patients, with non-smokers who initiated bupropion treatment losing an estimated 
$8.4 \mathrm{lbs}(95 \% \mathrm{CI}:-16.5,-0.3 ; p$-value $=0.041)$ compared to non-smoking fluoxetine users. Smokers who initiated bupropion treatment gained an estimated $14.2 \mathrm{lbs}(95 \% \mathrm{CI}: 3.4,24.9 ; p$-value $=0.001)$ compared to fluoxetine non-smokers. Note that the estimated weight change in the non-smoking bupropion users is similar in the ITT and PP analysis; however, smokers are estimated to gain about $11 \mathrm{lbs}$ more if treated continuously for two years compared to bupropion treated patients in the ITT analysis, most of whom did not complete two years of treatment (Table 2). Other antidepressant drug effects on body weight were smaller and not statistically significantly different than fluoxetine. There were an insufficient number of mirtazapine, duloxetine, and venlafaxine users to estimate two-year weight change for those drugs using our PP analyses.

Table 4. Estimated 2-year weight change (lbs) for users of the various drug groups compared to fluoxetine users based on the per-protocol analysis *.

\begin{tabular}{ccccccc}
\hline & \multicolumn{3}{c}{ Unweighted Analysis } & \multicolumn{3}{c}{ Weighted Analysis } \\
\hline & Estimate & $p$-value & $\mathbf{9 5 \%}$ CI & Estimate & $p$-value & $\mathbf{9 5 \%}$ CI \\
\hline Bupropion: non smoker & -7.6 & 0.049 & $(-15.2,0.0)$ & -8.4 & 0.041 & $(-16.5,-0.3)$ \\
Bupropion: smoker & 13.3 & 0.016 & $(2.5,24.1)$ & 14.2 & 0.001 & $(3.4,24.9)$ \\
Citalopram & 1.9 & 0.49 & $(-3.5,7.2)$ & 2.9 & 0.32 & $(-2.9,8.7)$ \\
Paroxetine & 0.9 & 0.86 & $(-9.2,11.0)$ & 0.3 & 0.96 & $(-10.5,11.1)$ \\
Sertraline & 2.3 & 0.53 & $(-5.0,9.6)$ & 5.5 & 0.17 & $(-2.4,13.4)$ \\
Trazodone & 0.9 & 0.88 & $(-11.4,13.3)$ & -0.1 & 0.99 & $(-13.2,13.0)$ \\
\hline
\end{tabular}

* Results in the left part of the table refer to an unweighted modelling that ignores selection bias, while the right side of the tables provides the inverse probability weighted estimation results. Omnibus $p$-values for the null hypothesis "all drugs have the same effect on weight change" were 0.21 (naive analysis) and 0.09 (IPW analysis). Analyses were adjusted for age, gender, baseline weight, smoking status, and active psychotherapy.

\section{Discussion}

In this large, population-based study of antidepressant use among patients with diagnosed depression, we found that bupropion was associated with significantly less weight gain than fluoxetine among non-smokers after two years follow-up, while there was no significant difference in weight gain between bupropion and fluoxetine among smokers. Bupropion has previously been associated with weight loss in short-term (one year or less duration) studies of both depressed and non-depressed individuals $[18,37]$. While the mechanism of the weight-reducing effect of bupropion has not been determined, it is suspected that the dopaminergic and noradrenergic effects of bupropion play important roles in the regulation of appetite, satiety, craving, and feeding behavior [38]. The only other antidepressant medication in our study that was associated with significant weight changes at two years was sertraline, which was associated with modest weight gain compared with fluoxetine. Only the findings for bupropion were robust when we considered those patients who had continued treatment with the medications for a full two years (our PP analysis). The results are consistent with a recent 12-month study conducted by Blumenthal and colleagues involving over 22,000 adults in a single health system in New England [24].

Our study is important because antidepressants are among the most commonly prescribed medications in the United States (10.4\% of adults) [9], and because there is currently no evidence to suggest that antidepressant medications differ in terms of their efficacy [26,27]. Given that bupropion is the only antidepressant associated with long-term weight loss, this medication should be the first-line drug of choice for all overweight and obese patients unless there are other existing contraindications such as a history of seizure disorder, anorexia nervosa or bulimia, or patients undergoing abrupt discontinuation of ethanol or sedatives including anticonvulsants, barbiturates, or benzodiazepines. All other antidepressant medications should be considered second-line pharmacological treatments for depression among overweight and obese patients.

Our prior research with this same population demonstrated that body weight was significantly associated with the choice of initial antidepressant medication, although the associations were 
weak [39]. Patients with lower BMIs were more likely to receive mirtazapine and those with higher BMIs were more likely to receive bupropion. The current study confirms that providers should strongly consider the selective prescribing of bupropion in patients who are overweight or obese. However, not all patients with depression are overweight or in need of weight loss, and, as a result, bupropion may be less appropriate for normal and underweight individuals who are nonsmokers. Similarly, in any patients who are underweight, it may be desirable to consider an antidepressant that is associated with weight gain, such as sertraline.

Our study has a number of limitations that should be mentioned. Our analysis was limited only to patients in the integrated group practice of a single large health plan; this form of analysis should be replicated in other systems. Second, in our PP analysis, we have a very small number of people actually completing the two years of treatment. We are reassured that the findings in this cohort are very similar to the larger group in the ITT analysis. However, the ITT analysis does include patients who initiated treatment but did not continue the antidepressant drug for the full two years, so the estimates of drug effects may be biased. We did not have enough patients receiving some antidepressant medications (mirtazapine, duloxetine, and venlafaxine) to estimate two year weight changes using both the ITT and PP methods. The changes in weight that we observed were clinically small for all but the bupropion users. Some second-generation antidepressants were not on the Group Health formulary and were thus excluded from our analyses (including escitalopram, fluvoxamine, and nefazodone) owing to low numbers of patients prescribed these drugs in our databases. While we excluded patients taking a number of other medications that might potentially influence the trajectory of body weight (antipsychotics, weight loss drugs), we did not look for or exclude patients who may have received metformin, lisdexamfetamine, topiramate, cyproheptadine, megestrol, or cannabinoid derivatives. Therefore our results should be interpreted with some caution as it is possible that we included some patients who received these medications while not accounting for their potential weight effects. Overall, we expect the effects of these drugs to have only a minor influence on our main result as most are rarely prescribed and have only a modest impact on weight.

In conclusion, we find that bupropion is the only antidepressant associated with long-term weight loss (although this effect is limited to non-smokers). Given similar efficacy for improvement in depressive symptoms across bupropion and other second-generation antidepressants, bupropion may be considered the first-line drug of choice for overweight and obese patients unless there are other existing contraindications.

Supplementary Materials: Supplementary materials can be found at http://www.mdpi.com/2077-0383/ 5/4/48/s1.

Acknowledgments: This project was funded under grant R01 MH083671 from the National Institute of Mental Health (NIMH) - Principal investigator: D. Arterburn. The authors of this report are responsible for its content.

Author Contributions: D.A., T.S., D.M.B., A.B., E.O.W., G.S., and S.H. conceived and designed the study; T.S., A.B., M.K.T., and S.H. prepared the databases; T.S., A.B., M.K.T., and S.H. analyzed the data; D.A., T.S., and S.H. wrote the paper; and all authors provided significant edits/revisions to the paper.

Conflicts of Interest: The authors declare no conflict of interest.

\section{Abbreviations}

The following abbreviations are used in this manuscript: BMI, body mass index; GH, Group Health; IGP, integrated group practice; IPW, inverse probability weighting; ITT, intent to treat; PP, per protocol; EHR, electronic health record

\section{References}

1. U.S. Department of Health and Human Services, Public Health Service, Office of the Surgeon General. The Surgeon General's Call to Action to Prevent and Decrease Overweight and Obesity; U.S. Department of Health and Human Services, Public Health Service, Office of the Surgeon General: Rockville, MD, USA, 2001.

2. Arterburn, D. Obesity. Clin. Evid. 2004, 11, 762-776. [PubMed] 
3. Arterburn, D.E.; McDonell, M.B.; Hedrick, S.C.; Diehr, P.; Fihn, S.D. Association of body weight with condition-specific quality of life in male veterans. Am. J. Med. 2004, 117, 738-746. [CrossRef] [PubMed]

4. Simon, G.E. Long-term prognosis of depression in primary care. Bull. World Health Organ. 2000, 78, 439-445. [PubMed]

5. Simon, G.E. Social and economic burden of mood disorders. Biol. Psychiatry 2003, 54, 208-215. [CrossRef]

6. Simon, G.E.; Chisholm, D.; Treglia, M.; Bushnell, D. Course of depression, health services costs, and work productivity in an international primary care study. Gen. Hosp. Psychiatry 2002, 24, 328-335. [CrossRef]

7. Simon, G.E.; Von Korff, M.; Lin, E. Clinical and functional outcomes of depression treatment in patients with and without chronic medical illness. Psychol. Med. 2005, 35, 271-279. [CrossRef] [PubMed]

8. Flegal, K.M.; Carroll, M.D.; Kit, B.K.; Ogden, C.L. Prevalence of obesity and trends in the distribution of body mass index among us adults, 1999-2010. JAMA 2012, 307, 491-497. [CrossRef] [PubMed]

9. Shim, R.S.; Baltrus, P.; Ye, J.; Rust, G. Prevalence, treatment, and control of depressive symptoms in the united states: Results from the national health and nutrition examination survey (nhanes), 2005-2008. J. Am. Board Fam. Med. 2011, 24, 33-38. [CrossRef] [PubMed]

10. Linde, J.A.; Jeffery, R.W.; Finch, E.A.; Simon, G.E.; Ludman, E.J.; Operskalski, B.H.; Ichikawa, L.; Rohde, P. Relation of body mass index to depression and weighing frequency in overweight women. Prev. Med. 2007, 45, 75-79. [CrossRef] [PubMed]

11. Simon, G.E.; Von Korff, M.; Saunders, K.; Miglioretti, D.L.; Crane, P.K.; van Belle, G.; Kessler, R.C. Association between obesity and psychiatric disorders in the us adult population. Arch. Gen. Psychiatry 2006, 63, 824-830. [CrossRef] [PubMed]

12. Heo, M.; Pietrobelli, A.; Fontaine, K.R.; Sirey, J.A.; Faith, M.S. Depressive mood and obesity in us adults: Comparison and moderation by sex, age, and race. Int. J. Obes. (Lond.) 2006, 30, 513-519. [CrossRef] [PubMed]

13. Ogden, C.L.; Carroll, M.D.; Curtin, L.R.; McDowell, M.A.; Tabak, C.J.; Flegal, K.M. Prevalence of overweight and obesity in the united states, 1999-2004. JAMA 2006, 295, 1549-1555. [CrossRef] [PubMed]

14. Flegal, K.M.; Carroll, M.D.; Kuczmarski, R.J.; Johnson, C.L. Overweight and obesity in the united states: Prevalence and trends, 1960-1994. Int. J. Obes. Relat. Metab. Disord. 1998, 22, 39-47. [CrossRef] [PubMed]

15. Paulose-Ram, R.; Safran, M.A.; Jonas, B.S.; Gu, Q.; Orwig, D. Trends in psychotropic medication use among U.S. Adults. Pharmacoepidemiol. Drug Saf. 2007, 16, 560-570. [CrossRef] [PubMed]

16. Keith, S.W.; Redden, D.T.; Katzmarzyk, P.T.; Boggiano, M.M.; Hanlon, E.C.; Benca, R.M.; Ruden, D.; Pietrobelli, A.; Barger, J.L.; Fontaine, K.R.; et al. Putative contributors to the secular increase in obesity: Exploring the roads less traveled. Int. J. Obes. (Lond.) 2006, 30, 1585-1594. [CrossRef] [PubMed]

17. Ricca, V.; Mannucci, E.; Di Bernardo, M.; Rizzello, S.M.; Cabras, P.L.; Rotella, C.M. Sertraline enhances the effects of cognitive-behavioral treatment on weight reduction of obese patients. J. Endocrinol. Investig. 1996, 19, 727-733. [CrossRef]

18. Serretti, A.; Mandelli, L. Antidepressants and body weight: A comprehensive review and meta-analysis. J. Clin. Psychiatry 2010, 71, 1259-1272. [CrossRef] [PubMed]

19. Aronne, L.J.; Segal, K.R. Weight gain in the treatment of mood disorders. J. Clin. Psychiatry 2003, 64 (Suppl. 8), 22-29. [PubMed]

20. Berkowitz, R.I.; Fabricatore, A.N. Obesity, psychiatric status, and psychiatric medications. Psychiatr. Clin. North Am. 2005, 28, 39-54. [CrossRef] [PubMed]

21. Deshmukh, R.; Franco, K. Managing weight gain as a side effect of antidepressant therapy. Cleve Clin. J. Med. 2003, 70. [CrossRef]

22. Kachur, S.G.; Hannan, C.L.; Ward, K.E. Antidepressant-induced weight gain. Med. Health R. I. 2005, 88, 359-361. [PubMed]

23. Schwartz, T.L.; Nihalani, N.; Jindal, S.; Virk, S.; Jones, N. Psychiatric medication-induced obesity: A review. Obes. Rev. 2004, 5, 115-121. [CrossRef] [PubMed]

24. Blumenthal, S.R.; Castro, V.M.; Clements, C.C.; Rosenfield, H.R.; Murphy, S.N.; Fava, M.; Weilburg, J.B.; Erb, J.L.; Churchill, S.E.; Kohane, I.S.; et al. An electronic health records study of long-term weight gain following antidepressant use. JAMA Psychiatry 2014, 71, 889-896. [CrossRef] [PubMed] 
25. Qaseem, A.; Snow, V.; Denberg, T.D.; Forciea, M.A.; Owens, D.K.; Clinical Efficacy Assessment Subcommittee of the American College of Physicians. Using second-generation antidepressants to treat depressive disorders: A clinical practice guideline from the american college of physicians. Ann. Intern. Med. 2008, 149, 725-733. [CrossRef] [PubMed]

26. Gartlehner, G.; Gaynes, B.N.; Hansen, R.A.; Thieda, P.; DeVeaugh-Geiss, A.; Krebs, E.E.; Moore, C.G.; Morgan, L.; Lohr, K.N. Comparative benefits and harms of second-generation antidepressants: Background paper for the american college of physicians. Ann. Intern. Med. 2008, 149, 734-750. [CrossRef] [PubMed]

27. Cipriani, A.; Furukawa, T.A.; Salanti, G.; Geddes, J.R.; Higgins, J.P.; Churchill, R.; Watanabe, N.; Nakagawa, A.; Omori, I.M.; McGuire, H.; et al. Comparative efficacy and acceptability of 12 new-generation antidepressants: A multiple-treatments meta-analysis. Lancet 2009, 373, 746-758. [CrossRef]

28. Aubin, H.J.; Farley, A.; Lycett, D.; Lahmek, P.; Aveyard, P. Weight gain in smokers after quitting cigarettes: Meta-analysis. BMJ 2012, 345, e4439. [CrossRef] [PubMed]

29. Saunders, K.D.R.; Stergachis, A. Group health cooperative. In Pharmacoepidemiology, 4th ed.; BL, S., Ed.; John Wiley and Sons: West Sussez, UK, 2005; pp. 223-239.

30. Boudreau, D.M.; Daling, J.R.; Malone, K.E.; Gardner, J.S.; Blough, D.K.; Heckbert, S.R. A validation study of patient interview data and pharmacy records for antihypertensive, statin, and antidepressant medication use among older women. Am. J. Epidemiol. 2004, 159, 308-317. [CrossRef] [PubMed]

31. Buist, D.S.; LaCroix, A.Z.; Brenneman, S.K.; Abbott, T., 3rd. A population-based osteoporosis screening program: Who does not participate, and what are the consequences? J. Am. Geriatr. Soc. 2004, 52, 1130-1137. [CrossRef] [PubMed]

32. Arterburn, D. Bariatric surgery. BMJ 2008, 337, a755. [CrossRef] [PubMed]

33. Seaman, S.R.; White, I.R. Review of inverse probability weighting for dealing with missing data. Stat. Methods Med. Res. 2013, 22, 278-295. [CrossRef] [PubMed]

34. Robbins, J.M.; Hernan, M.A.; Brumback, B. Marginal structural models and causal inference in epidemiology. Epidemiology 2000, 11, 550-560. [CrossRef]

35. Cole, S.R.; Hernan, M.A. Constructing inverse probability weights for marginal structural models. Am. J. Epidemiol. 2008, 168, 656-664. [CrossRef] [PubMed]

36. Follmann, D.; Proschan, M.; Leifer, E. Multiple outputation: Inference for complex clustered data by averaging analyses from independent data. Biometrics 2003, 59, 420-429. [CrossRef] [PubMed]

37. Jain, A.K.; Kaplan, R.A.; Gadde, K.M.; Wadden, T.A.; Allison, D.B.; Brewer, E.R.; Leadbetter, R.A.; Richard, N.; Haight, B.; Jamerson, B.D.; et al. Bupropion SR vs. placebo for weight loss in obese patients with depressive symptoms. Obes. Res. 2002, 10, 1049-1056. [CrossRef] [PubMed]

38. Stahl, S.M.; Pradko, J.F.; Haight, B.R.; Modell, J.G.; Rockett, C.B.; Learned-Coughlin, S. A review of the neuropharmacology of bupropion, a dual norepinephrine and dopamine reuptake inhibitor. Prim. Care Companion J. Clin. Psychiatry 2004, 6, 159-166. [CrossRef] [PubMed]

39. Boudreau, D.M.; Arterburn, D.; Bogart, A.; Haneuse, S.; Theis, M.K.; Westbrook, E.; Simon, G. Influence of body mass index on the choice of therapy for depression and follow-up care. Obesity (Silver Spring) 2013, 21, E303-E313. [CrossRef] [PubMed]

(C) 2016 by the authors; licensee MDPI, Basel, Switzerland. This article is an open access article distributed under the terms and conditions of the Creative Commons Attribution (CC-BY) license (http://creativecommons.org/licenses/by/4.0/). 